\title{
Seeing how we smell
}

\author{
Helene Benveniste, ${ }^{1}$ Yuri Lazebnik, ${ }^{2}$ and Nora D. Volkow ${ }^{3}$ \\ 'Department of Anesthesiology, Yale School of Medicine, New Haven, Connecticut, USA. ${ }^{2}$ Lerna Consulting, LLC, New Haven, Connecticut, USA. ${ }^{3}$ Laboratory for Neuroimaging, National Institute on Alcohol \\ Abuse and Alcoholism (NIAAA), NIH, Bethesda, Maryland, USA.
}

\begin{abstract}
PET allows noninvasive imaging of a variety of events in the body, including the activity of neuronal circuits in the brain that are involved in cognition and behaviors, by using radiotracers that detect relevant biological reactions. A major impediment to expanding PET applications to study the brain has been the lack of radiotracers that can identify and measure specific types of neurons or glial cells. In this issue of the $J C I$, Van de Bittner and colleagues describe a promising step toward solving this problem by identifying and describing a radiotracer, ["C]CV1-57, that appears to specifically label olfactory sensory neurons (OSNs), which are essential for olfaction (Figure 1). This tracer, if its specificity is confirmed, has the potential to become a prototype for future radiotracers that can identify other neuronal cell types and would allow visualization and in-depth characterization of these neurons and their genesis.
\end{abstract}

brain has been the lack of radiotracers that can identify and measure specific types of neurons or glial cells.

In this issue, Van de Bittner et al. describe a promising step toward solving this problem (5). Specifically, the authors discovered and characterized a new PET radiotracer, $\left[{ }^{11} \mathrm{C}\right] \mathrm{GV} 1-57$, which, they conclude, labels the mature olfactory sensory neurons (OSNs) of the olfactory epithelium, the tissue involved in recognizing smells (Figure 1). The olfactory epithelium consists of three cell types: OSNs, sensory-supporting cells, and germ cells (or undifferentiated basal cells) (6). The OSNs are true neurons (Golgi type I) that have the unique property of being continuously renewed by neurogenesis, a process involving the cycle of birth, maturation, and death (7). As a consequence of continuous neurogenesis, the olfactory epithelium contains OSNs at different stages of maturation, while the number of live OSNs at any given time is a result of the balance between the rates of their generation and death (7).

By studying the structure-function relationship of a class of radiotracers, Van de Bittner and colleagues determined that $\left[{ }^{11} \mathrm{C}\right] \mathrm{GV} 1-57$ is specifically taken up by the olfactory epithelium of rodents and nonhuman primates. The authors then identified OSNs as the cells targeted by $\left[{ }^{11} \mathrm{C}\right] \mathrm{GV} 1-57$ by surgically removing the olfactory bulbs in rats, a procedure that causes complete degeneration of mature OSNs but does not affect the other two cell types of the olfactory epithelium (8). $\left[{ }^{11} \mathrm{C}\right] \mathrm{GV} 1-57$ binding correlated with the number of mature OSNs (quantified via the OSN-specific olfactory marker protein) after surgery, indicating that the tracer targets mature OSNs. Other cell types, such as immature OSNs and sustentacular cells, were not associated with changes in $\left.{ }^{[11} \mathrm{C}\right] \mathrm{GV} 1-57$ binding after bulbectomy. However, the surgery only partially decreased $\left[{ }^{11} \mathrm{C}\right] \mathrm{GV} 1-57$ binding, and future studies will therefore need to establish whether the remaining binding 


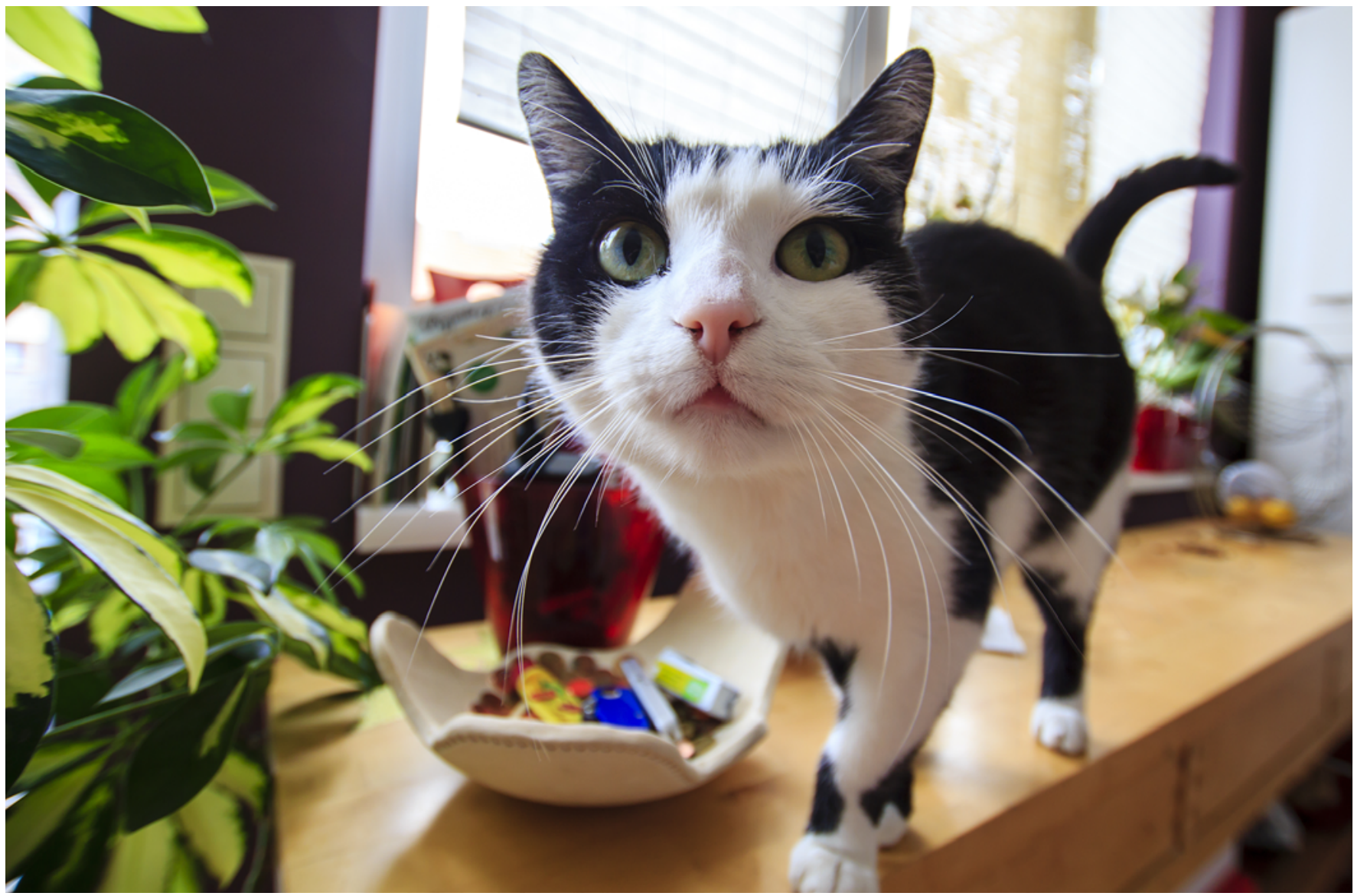

Figure 1. In this issue, Van de Bittner and colleagues describe a PET radiotracer that has the potential to allow visualization of OSN formation and turnover.

is nonspecific or due to a particular cell type other than mature OSNs.

There are two main implications of the discovery of this new radiotracer, should its specificity to mature OSNs be confirmed. First, it would provide proof of principle that a specific neuronal type can be visualized in the body. Second, this molecule would provide a tool for studying neurogenesis in the brain in animals and, potentially, in humans.

\section{Detecting specific neuronal types}

To our knowledge, $\left[{ }^{11} \mathrm{C}\right] \mathrm{GV} 1-57$ is the first PET radiotracer that can identify and quantify a specific neuronal subtype, irrespective of the neuron's functional state. Previously developed PET tracers have been considered to be "blind" to the cell type, because they bind receptors, transporters, or enzymes, the concentration and functions of which are associated with the activity of more than one type of neuron. For example, radiotracers for dopa- mine transporters were initially developed as biomarkers of dopamine neurons (9, 10), but subsequent studies revealed that the expression of these transporters in the membrane is dynamic and varies as a function of the extracellular dopamine levels (upregulating when dopamine levels are high and downregulating when levels are low) (11). Dopamine transporter expression also depends on circadian rhythms (12). Similarly, up- and downregulation of serotonin and norepinephrine transporters present in the membrane preclude their use as markers of noradrenergic and serotonergic neuronal populations. L-3,4-dihydroxyphenylalanine (L-DOPA), which labels tyrosine hydroxylase (an enzyme involved in dopamine synthesis), was proposed as a marker of dopamine neurons, but its concentration also varies depending on the neurotransmitter demand (13). Various other ligands, such as those that bind to dopamine D2 and D3 receptors, which can be labeled by $\left[{ }^{11} \mathrm{C}\right]$-raclopride and $\left[{ }^{18} \mathrm{~F}\right]$-fallypride; $\mu$ opiate receptors, which are identified by $\left[{ }^{11} \mathrm{C}\right]$ carfentanil (14); or monamine oxidase $\mathrm{A}$, which is targeted by $\left[{ }^{11} \mathrm{C}\right]$-clorgyline $(15,16)$, are all expressed in multiple neuronal types and some also in glial cells.

The discovery that a radiotracer can target a specific type of neuron is particularly remarkable, because it provides a proof of principle that cell types in the brain can be noninvasively identified. This proof implies the possibility that tracers for other neuronal types can also be developed. Time will tell whether these tracers will be discovered by the approach used by Van de Bittner and colleagues or otherwise, but in any case, a pioneering finding like theirs often opens a floodgate of further discoveries and their practical applications. The resulting noninvasive imaging approaches would be very timely for the BRAIN (Brain Research through Advancing Innovative Neurotechnologies) initiative, which is aimed at "identifying the diversity of brain cells" (17), a goal that would be difficult to reach without new tools for classifying, 
characterizing, and manipulating specific cell types in animal models and in humans.

Tracers like $\left[{ }^{11} \mathrm{C}\right] \mathrm{GV} 1-57$ and screening approaches to find them would be of great benefit, as the taxonomy of many neuronal types have yet to be defined, and current classifications based on anatomy, physiology, and even genetic expression patterns may still prove to be insufficient to classify the $1 \times 10^{11}$ neurons in the human brain, of which 145 types have been recognized so far (18). How incomplete this list might be is suggested by the finding that the mere 302 neurons of the nematode Caenorhabditis elegans fall into 118 classes (19).

\section{Visualizing neurogenesis}

Because OSNs undergo neurogenesis, a poorly understood process by which new neurons are generated throughout life (7), $\left[{ }^{11} \mathrm{C}\right] \mathrm{GV} 1-57$ may serve as a prototype tool to study how new neurons are generated in the brain. Van de Bittner et al. tested this possibility by injuring the olfactory epithelium with zinc sulfate irrigation in mice and then used $\left[{ }^{11} \mathrm{C}\right] \mathrm{GV} 1-57$ to visualize OSN death two to three days after injury and OSN repopulation over the subsequent two months. These experiments showed that the net gain of mature OSNs after injury might reflect neurogenesis, neuron maturation, and/or changes in neuron death, as all of these processes can ultimately influence the total mature OSN population. These observations open the possibility of testing whether $\left[{ }^{11} \mathrm{C}\right]$ GV1-57 or its successors can be used for monitoring and measuring neurogenesis in the human brain. Because the loss of smell is associated with or precedes several neurodegenerative diseases (20), radiotracers like $\left[{ }^{11} \mathrm{C}\right] \mathrm{GV} 1-57$ may be used diagnostically as early indicators of Alzheimer's disease and, consequently, for testing therapeutic interventions aimed at promoting neurogenesis.

\section{Summary}

$\left.{ }^{[11} \mathrm{C}\right] \mathrm{GV} 1-57$ is the first reported PET radiotracer that can detect a specific neuronal type (Golgi type I); thus, the identification of this radiotracer provides hope that future targets for identifying cell diversity in the living brain can be found. As $\left[{ }^{11} \mathrm{C}\right] \mathrm{CV} 1-57$ happens to detect neurons continuously produced by neurogenesis in the brain, this tracer and, likely, its successors have the potential to serve as biomarkers for trauma, toxicity, and neurodegeneration, as well as reporters for therapeutic development.

Address correspondence to: Helene Benveniste, Department of Anesthesiology, Yale School of Medicine, New Haven, Connecticut 11794, USA. Phone: 203.737.6934;

E-mail: helene.benveniste@yale.edu.

1. Fowler JS, Ido T. Initial and subsequent approach for the synthesis of 18FDG. Semin Nucl Med. 2002;32(1):6-12.

2. Klunk WE, et al. Imaging brain amyloid in Alzheimer's disease with Pittsburgh Compound-B. Ann Neurol. 2004;55(3):306-319.

3. Okamura N, Harada R, Furumoto S, Arai H, Yanai K, Kudo Y. Tau PET imaging in Alzheimer's disease. Curr Neurol Neurosci Rep. 2014;14(11):500.

4. Schweitzer PJ, Fallon BA, Mann JJ, Kumar JS. PET tracers for the peripheral benzodiazepine receptor and uses thereof. Drug Discov Today. 2010;15(21-22):933-942.

5. Van de Bittner GC, et al. Nasal neuron PET imaging quantifies neuron generation and degeneration. JClin Invest. 2017;127(2):681-694.

6. Farbman AI. Olfactory neurogenesis: genetic or environmental controls? Trends Neurosci. 1990;13(9):362-365.
7. Brann JH, Firestein SJ. A lifetime of neurogenesis in the olfactory system. Front Neurosci. 2014;8:182.

8. Costanzo RM, Graziadei PP. A quantitative analysis of changes in the olfactory epithelium following bulbectomy in hamster. J Comp Neurol. 1983;215(4):370-381

9. Fowler JS, et al. Measuring dopamine transporter occupancy by cocaine in vivo: radiotracer considerations. Synapse. 1998;28(2):111-116.

10. Piccini PP. Dopamine transporter: basic aspects and neuroimaging. Mov Disord. 2003;18(suppl 7):S3-S8.

11. Volkow ND, et al. Recovery of dopamine transporters with methamphetamine detoxification is not linked to changes in dopamine release. Neuroimage. 2015;121:20-28.

12. Ferris MJ, et al. Dopamine transporters govern diurnal variation in extracellular dopamine tone. Proc Natl Acad Sci U S A. 2014;111(26):E2751-E2759.

13. Solanki RR, Scholl JL, Watt MJ, Renner KJ, Forster GL. Amphetamine withdrawal differentially increases the expression of organic cation transporter 3 and serotonin transporter in limbic brain regions. J Exp Neurosci. 2016;10:93-100.

14. Frost JJ, et al. Multicompartmental analysis of [11C]-carfentanil binding to opiate receptors in humans measured by positron emission tomography. JCereb Blood Flow Metab. 1989;9(3):398-409.

15. Fowler JS, et al. Species differences in [11C] clorgyline binding in brain. Nucl Med Biol. 2001;28(7):779-785.

16. Fowler JS, et al. Mapping human brain monoamine oxidase A and B with 11C-labeled suicide inactivators and PET. Science. 1987;235(4787):481-485.

17. Jorgenson LA, et al. The BRAIN Initiative: developing technology to catalyse neuroscience discovery. Philos Trans R Soc Lond B Biol Sci. 2015;370(1668):20140164.

18. Vickaryous MK, Hall BK. Human cell type diversity, evolution, development, and classification with special reference to cells derived from the neural crest. Biol Rev Camb Philos Soc. 2006;81(3):425-455.

19. Hobert O, Glenwinkel L, White J. Revisiting neuronal cell type classification in Caenorhabditis elegans. Curr Biol. 2016;26(22):R1197-R1203.

20. Hawkes C. Olfaction in neurodegenerative disorder. Adv Otorhinolaryngol. 2006;63:133-151. 